\title{
Image quality and dose reduction in sinus computed tomography using iterative reconstruction: a cadaver study*
}

\author{
Adam J. Kimple', Stanley W. McClurg', Benjamin Y. Huang², \\ Satyan B. Sreenath', Benjamin W. McClintock², Mohamed Tomoum³, \\ Feng-Chang Lin ${ }^{4}$, Charles S. Ebert ${ }^{1}$, Brent A. Senior ${ }^{1,5}$ \\ 1 Department of Otolaryngology - Head and Neck Surgery, University of North Carolina at \\ Chapel Hill, NC, USA \\ ${ }^{2}$ Department of Radiology, University of North Carolina at Chapel Hill, NC, USA \\ ${ }^{3}$ Department of Otolaryngology - Head and Neck Surgery, Tanta University, Tanta, Egypt \\ ${ }^{4}$ Carolina Translational and Clinical Science Institute, NC, USA \\ ${ }^{5}$ Department of Neurosurgery, University of North Carolina at Chapel Hill, NC, USA
}

Rhinology Online, Vol 1: $45-49,2018$
http://doi.org/10.4193/RHINOL/18.015

*Received for publication:

March 7, 2018

Accepted: April 21, 2018

Published: May 8, 2018

\begin{abstract}
Introduction: Concerns about radiation dose in computed tomography (CT) imaging have renewed interest in iterative reconstruction (IR), a technique which has the potential to produce images with less noise at lower radiation doses than traditional filtered back projection (FBP). This study aimed to assess whether application of IR could provide comparable quality sinus CT images to FBP at lower kilovolt $(\mathrm{kV})$ and milliamp $(\mathrm{mA})$ settings, and to establish optimal scan settings for sinus imaging.
\end{abstract}

Methods: 30 sinus CT scans were performed on 5 cadaver heads at two $\mathrm{kV}$ setting and three $\mathrm{mA}$ settings. Each scan was reconstructed using FBP and 3 IR settings, yielding a total of 120 image series. Each image set was blinded and randomly reviewed by 3 rhinologists and 2 neuroradiologists. Using a 5-point Likert scale, 16 anatomical landmarks, were graded with respect to image quality. Data were assessed with respect to dose and IR settings using statistical analysis.

Results: Higher kV and mAs settings produced significantly higher quality images for structure identification across all 16 landmarks; however, the suitability for surgery did not increase in a linear fashion and plateaued by a total radiation dose of 0.1201 $\mathrm{mSv}$. IR algorithm did not provide a benefit in the overall score of scans at a fixed $\mathrm{kV}$ and $\mathrm{mAs}$.

Conclusions: Identification of structures in sinus CT imaging significantly correlates with the $\mathrm{kV}$ and $\mathrm{mA}$ and overall dose of radiation; however, IR did not provide additional benefit in the image quality. Blinded reviewers unanimously agreed that scans obtained at $100 \mathrm{mV}$ and $120 \mathrm{~mA}$ were acceptable for surgery independent of IR.

Key words: sinus anatomy, computed tomography, radiology, imaging, computer assisted surgery

\section{Introduction}

Rhinosinusitis has a tremendous worldwide burden and is estimated to affect $16 \%$ of the US adult population annually ${ }^{(1)}$. Additionally, quality of life studies have demonstrated patients with rhinosinusitis have a worse quality of life than patients with heart failure, chronic obstructive pulmonary disorder or Parkinson's disease ${ }^{(2)}$. While the underlying pathophysiology is complex, rhinosinusitis involves an imbalance of the immune system, sinonasal microbiome, and mucociliary clearance ${ }^{(3)}$. Rhinosinutitis in adults is defined as inflammation of the nose and paranasal sinuses associated with symptoms such as nasal obstruction or nasal drainage and either endoscopic evidence of disease or computed tomography (CT) demonstrating mucosal changes $^{(4)}$.

When assessing the benefits of a CT scan for evaluation of sino- 
nasal disease, the inherent risk of associated radiation exposure must be considered. While side effects of radiation exposure are difficult to completely characterize and quantify, physicians and patients should be cognizant of increased risk of cataracts and malignancies with radiation exposure ${ }^{(5-7)}$. The potential adverse effects from CT scans are especially concerning for pediatric patients and patients with chronic diseases that may require serial CT scans throughout their life.

After the atomic era during World War II, the Nuclear Regulatory Commission adopted stringent safeguards to protect civilians and nuclear workers. This led to the implementation of the ALARA (As Low as Reasonably Achievable) safety protocol for radiation exposure, which included medical diagnostic imaging. The goal of the ALARA protocol is to obtain CT scans with the lowest possible kilovoltage $(\mathrm{kV})$ and milliamps $(\mathrm{mA})$ that are necessary for appropriate and adequate evaluation. Iterative Reconstruction (IR) is a reconstruction algorithm that has been proposed as a solution to increase the relative clarity of CT scans, and furthermore allow the $\mathrm{kV}$ and $\mathrm{mA}$ to be subsequently reduced $^{(8)}$. Various proprietary iterative reconstructions are available and come packaged with the acquisition devices. Sinogram Affirmed Iterative Reconstruction (SAFIRE) is an IR protocol developed by Siemens (Siemens Medical Solutions, Erlangen, Germany), GE Healthcare, Philips, and Toshiba also have similar proprietary software. In general, in IR software, the user determines the iteration strength and overall amount of noise reduction. IR has the potential to produce low noise images with less radiation than traditional filtered back projection (FBP) reconstructed images. These benefits have been demonstrated in gelatin filled skulls ${ }^{(9)}$ but not in actual skulls with human tissue. Our use of cadaver heads, allowed control of kV, $\mathrm{mA}$ and IR setting without concern of radiation exposure and then by using blinded reviewers we were able to assess the image clarity with regards to specific structures as well as overall image suability for surgery.

\section{Materials and methods}

Five cadaver heads underwent $\mathrm{CT}$ imaging under varying kilovoltage $(\mathrm{kV})$ and milliamperage $(\mathrm{mA})$ settings on a 128-channel CT scanner (Somatom Definition, Siemens Healthcare, Erlangen, Germany). These images were then reconstructed using FBP and 3 IR settings. Data was collected using 100 or 120 $\mathrm{kV}$ and 30,60 or $120 \mathrm{~mA}$, resulting in a total of 6 unique dose settings. All other acquisition parameters were held constant (collimator width $=0.6 \mathrm{~mm}$, pitch factor $=0.8$, exposure time $=$ $1 \mathrm{~s})$. All image sets were reconstructed using traditional FBP or Sinogram Affirmed Iterative Reconstruction (SAFIRE, Siemens Healthcare, Erlangen, Germany), at settings of 1, 3 and 5. A total of 120 reconstructed $\mathrm{CT}$ image sets were therefore obtained. Each image set included $0.75 \mathrm{~mm}$ axial images reconstructed using a soft tissue (H40s) kernel and $2 \mathrm{~mm}$ axial, sagittal, and
Table 1. 5-point Likert scale for evaluation of CT scans.

\begin{tabular}{|cl|}
\hline Score & \multicolumn{1}{c}{ Description } \\
\hline 1 & structure is exceptionally well-visualized \\
\hline 2 & structure is easily visualized \\
\hline 3 & structure is indeterminate \\
\hline 4 & structure is poorly visualized \\
\hline 5 & structure is not able to be visualized \\
\hline
\end{tabular}

Table 2. Anatomical features and other characteristics that were evaluated by the blinded reviewers.

\begin{tabular}{l} 
Items Evaluated \\
maxillary sinuses \\
osteomeatal complex \\
frontal sinus \\
frontal recesses \\
anterior ethmoidal air cells \\
posterior ethmoidal air cells \\
sphenoethmoidal recess and sphenoid ostium \\
\hline sphenoid sinus and intersinus septum \\
\hline cribriform plate \\
\hline lamina papyracea \\
\hline Path of optic nerves \\
\hline Path of Internal Carotid Arteries \\
\hline Extraoccular Muscles \\
\hline Orbital Globes \\
\hline Anterior ethmoid artery \\
\hline Posterior ethmoid artery \\
\hline Overall Quality of Image \\
Is this a suitable image set for performing surgery? (Y/N) \\
\hline
\end{tabular}

coronal images reconstructed using a bone (H70h) kernel. These scans were then independently reviewed in a randomized and blinded fashion by 3 rhinologists (BAS, CES, and SWM) and 2 neuroradiologists (BYH and BWM).

Using a 5-point Likert scale (Table 1), visualization of 16 anatomical landmarks as well as overall image quality were graded (Table 2). The scale utilized was as follows: 1 = structure is exceptionally well visualized, 2 = structure is easily visualized, 3 $=$ structure is indeterminate, $4=$ structure is poorly visualized, $5=$ structure is not able to be visualized. Landmarks included the maxillary sinuses, ostio-meatal complexes (OMC), frontal sinuses, frontal recesses, anterior ethmoid sinuses, posterior ethmoid sinuses, spheno-ethmoidal recess/sphenoid ostia, sphenoid sinuses/inter-sinus septum, cribriform plates, lamina papyraceas, optic nerves, internal carotid arteries, extra-ocular muscles, orbital globes, anterior ethmoidal arteries, and posterior ethmoidal arteries. Each rater determined an "overall quality 


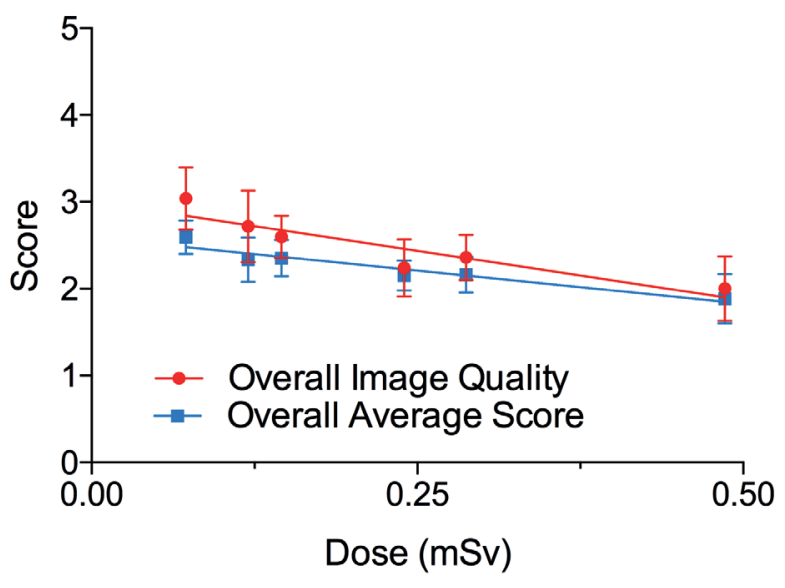

Figure 1. Overall image quality (red circles) and overall average (blue squares) score improved with increased radiation.

of image" for each scan on the same 5-point Likert scale and in a binary fashion determined if the scan was suitable for surgery. A separate mathematical average of all landmarks and the "overall quality of image" was calculated for each scan. Data were assessed with respect to dose and IR settings via ANOVA using a pvalue of 0.05 . To assess if this instrument had reliability between evaluators, we calculated an interclass correlation coefficient ${ }^{(10)}$.

\section{Results}

The interclass correlation coefficient was calculated across all structures to be 0.56 , indicating our instrument has "fair" agreement between evaluators. 10

The radiation dose in $\mathrm{mSv}$ for each of the 5 cadaver heads was calculated at each $\mathrm{kV}$ and $\mathrm{mAs}$ setting using a factor of 0.0021 $\mathrm{mSv} \mathrm{mGy}^{-1} \mathrm{~cm}^{-1}{ }^{111}$. As IR algorithms are mathematical refinements, they do not affect the $\mathrm{kV}, \mathrm{mAs}$ or $\mathrm{mSv}$ of a given scan. As dose of radiation increased, the average of all scores decreased, as did the overall image quality as determined by the evaluators. The average score of the scan improved from 3.04 at 0.07224 $\mathrm{mSv}(100 \mathrm{kV}, 30 \mathrm{~mA})$ to 2.00 at $0.48594 \mathrm{mSv}(120 \mathrm{kV}, 120 \mathrm{~mA})$. The "overall image quality" score improved from 2.5929 at $0.07224 \mathrm{mSv}(100 \mathrm{kV}, 30 \mathrm{~mA})$ to 1.8847 at $0.48594 \mathrm{mSv}(120 \mathrm{kV}$, $120 \mathrm{~mA}$; Figure 1).

We used linear regression to calculate the slope for the change in average score as radiation dose increased. A negative slope was obtained for all 16 anatomic sites (Figure 2); however, 95\% confidence intervals for both the sphenoidethmoid recess \& sphenoid ostium as well as the posterior ethmoid crossed zero indicating that the apparent improvement seen with increasing radiation dose was not statistically significant for these subsites (Figure 2A \& 2D).

At a highest dose setting of $120 \mathrm{kV}$ and $120 \mathrm{~mA}$ (0.48594 mSv) the sinuses tended to have better scores, while orbital structure and vascular structure had worse score (Figure 3). The structures
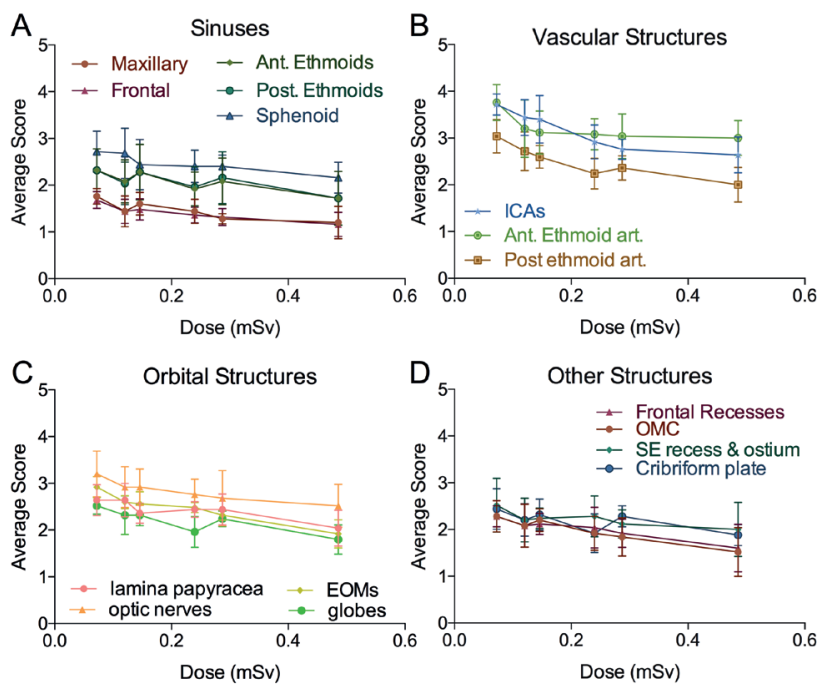

Figure 2. Average scores increased for all examined structures with increased radiation; however, vascular structure improved the most. (A) Modest improvement was noted in visualization of the sinuses. Over the range of settings an improvement of 0.568 was noted. (B) Vascular structures were particularly improved with increasing radiation. An improvement of 0.96 was noted from the lowest to highest settings. (C) Orbital structures and (D) other structures also improved with increased ionizing radiation by 0.75 and 0.63 respectively.

that were most difficult to visualize (score $>2.0$ ) at the highest setting ( $120 \mathrm{kV}$ and $120 \mathrm{mAs}$ ) were the posterior ethmoid artery, extraocular muscles (EOM), internal carotid artery, cribriform plate, and optic nerve (Figure 3).

In a binary fashion, each evaluator determined if scans were suitable for surgery. Using this endpoint, the scans obtained at $0.4859 \mathrm{mSv}(120 \mathrm{kV}, 120 \mathrm{~mA})$ and $0.2873 \mathrm{mSv}(100 \mathrm{kV}, 120 \mathrm{~mA})$ were unanimously suitable for surgery (Figure 4). Evaluators reported that $92 \%, 96 \%$, and $88 \%$ of the scans obtained at $0.2398 \mathrm{mSv}(120 \mathrm{kV}, 60 \mathrm{~mA}), 0.1457 \mathrm{mSv}(100 \mathrm{kV}, 60 \mathrm{~mA})$ and $0.1201 \mathrm{mSv}(120 \mathrm{kV}, 30 \mathrm{~mA}$ ) were suitable for surgery, respectively. Using a one-way ANOVA analysis, these differences were not statistically significant $(p>0.05)$ from results obtained at the highest setting, $0.4859 \mathrm{mSv}(120 \mathrm{kV}, 120 \mathrm{~mA})$. Scans obtained at the lowest energy setting $0.07224 \mathrm{mSv}(100 \mathrm{kV}, 30 \mathrm{~mA})$ were noted to be suitable for surgery in only $72 \%$ of cases, which was significantly worse than scans obtained at the highest settings, $0.4859 \mathrm{mSv}(120 \mathrm{kV}, 120 \mathrm{~mA})(\mathrm{p}<0.05)$.

Iterative reconstruction settings of 1, 3, and 5 were applied across all $\mathrm{kV}$ and $\mathrm{mAs}$ settings used (Figure 5). While again we saw significant improvement in the quality of scans with increasing radiation dose, there was no change in overall quality based on iterative reconstruction settings at any of the settings (Figure 5; $\mathrm{p}=0.8105$ ).

Various structures had different characteristics when evaluated (i.e. vascular structure had lower scores in general); therefore, we 


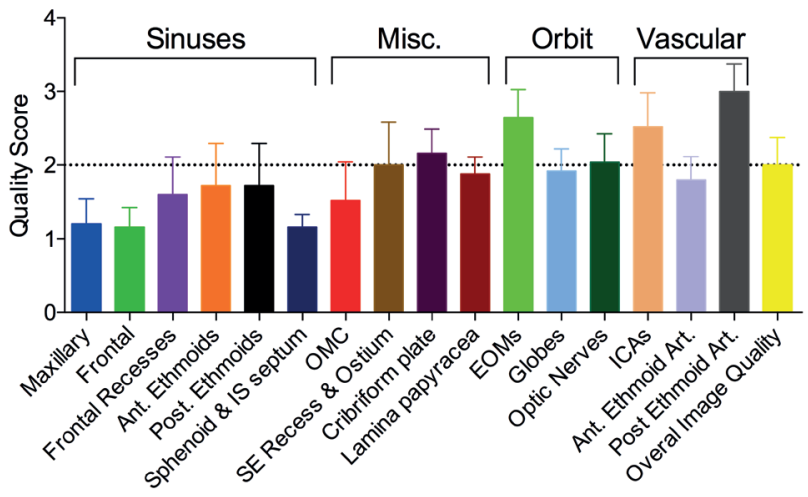

Figure 3. Sinuses and sinus landmarks had the best quality scores while orbital and vascular structures tended to have lower scores at setting of $120 \mathrm{kV}$ and $120 \mathrm{~mA}$. The posterior ethmoid artery, extraocular muscles (EOM), internal carotid artery, cribriform plate, and optic nerve had the lowest quality scores.

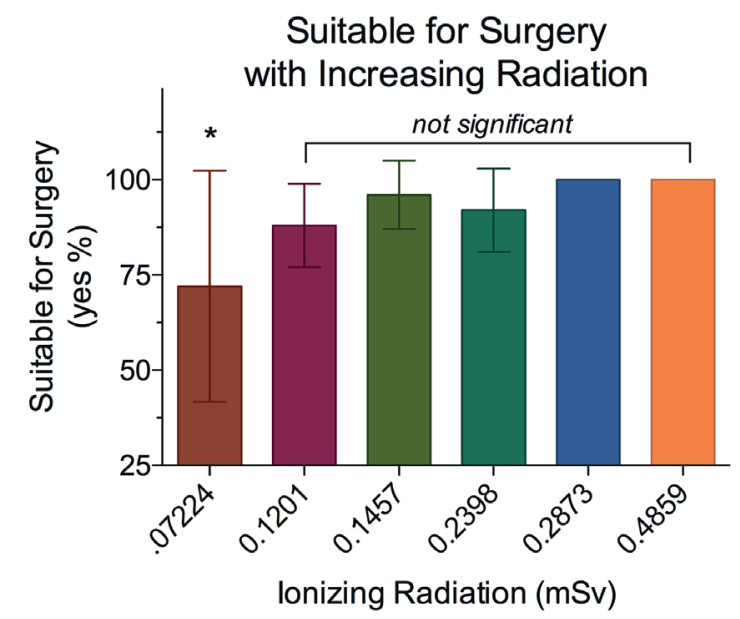

Figure 4. At the highest two radiation levels, the evaluators unanimously determined the scans were suitable for surgery. The scan obtained at $0.07224 \mathrm{mSv}(100 \mathrm{kV}, 30 \mathrm{~mA})$ was significantly inferior to the scans obtained at $0.4859(120 \mathrm{kV}, 120 \mathrm{~mA})$. The intermediate scans were statistically equivalent to the $0.4859(120 \mathrm{kV}, 120 \mathrm{~mA}) \mathrm{scan}$.

evaluated the effect of iterative reconstruction algorithms on each structure at low energy settings $0.07224 \mathrm{mSv}(100 \mathrm{kV}, 30$ $\mathrm{mA}$ ) and at high-energy settings $0.4859 \mathrm{mSv}(120 \mathrm{kV}, 120 \mathrm{~mA}$; $)$. The only individual factor that improved significantly with iterative reconstruction was the cribriform and only when using low energy setting $0.07224 \mathrm{mSv}(100 \mathrm{kV}, 30 \mathrm{~mA})$. IR did not improve any scores at $0.4859 \mathrm{mSv}(120 \mathrm{kV}, 120 \mathrm{~mA})$

\section{Discussion}

Computed tomography is critical to evaluating and treating chronic sinusitis. The American Academy of Otolaryngology - Head and Neck Surgery developed a clinical consensus statement that recommends CT scans for surgical planning, treatment of complications of sinusitis and for evaluation of

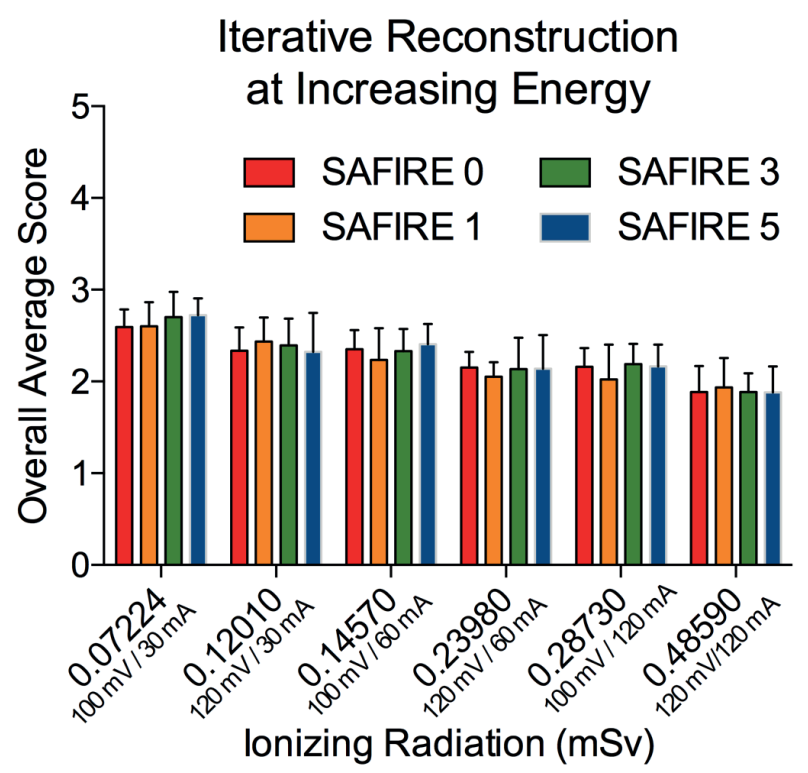

Figure 5. Iterative reconstruction did not improve the overall average score. Baseline scores without iterative reconstruction (red) are shown for all settings tested. IR setting of 1 (orange), 3 (green) and 5 (blue) did not improve the overall average score.

sinonasal tumors ${ }^{(12)}$. Additionally, physicians' use CT scans to provide additional information regarding disease severity and treatment response when endoscopic appearances do not appropriately correlate with perceived disease severity. The CT scan has wide utility; however, as clinicians we have a responsibility to minimize the potential harm from radiation exposure. $A$ study varying $\mathrm{kV}$ and $\mathrm{mA}$ over a wide degree would be unethical in humans because of the radiation exposure; however, using human cadaver heads allowed us to vary these levels without concern. Since we used a clinical CT scanner and the electrons passed through human bone and soft tissue, we expect our results would be representative of imaging performed in living patients. While increasing doses of radiation increased the quality of CT scans (Figures 1-3), we noted a threshold where additional radiation did not increase the suitability of a scan for surgery (Figure 4). All scans with a dose above $0.1201 \mathrm{mSv}$ (120 $\mathrm{kV}, 30 \mathrm{~mA}$ ) to $0.4859 \mathrm{mSv}(120 \mathrm{kV}, 120 \mathrm{~mA}$ ) were statistically equivalent in their suitability for surgery, regardless of reconstruction technique (Figure 4), although it should be noted that only at the 0.4859 and $(120 \mathrm{kV}, 120 \mathrm{~mA})$ and $0.2873 \mathrm{mSv}(100$ $\mathrm{kV}, 120 \mathrm{~mA}$ ) settings were unanimously deemed suitable for surgical planning. Approximately a 50\% reduction in radiation exposure was possible while maintaining a high quality image that was unanimously deemed suitable for surgery. Clinician should consider working with their radiologist to determine if $\mathrm{kV}$ setting can be lowered on their own equipment while maintain a high quality image.

Our initial hypothesis was that IR would allow us to trade com- 
putational time for less radiation exposure while maintaining image quality for surgery. We did not observe this and it was noted that multiple rounds of iterative reconstruction produced no improvement in scan quality when compared to traditional FBP approaches (Figure 5).

While many machines have automated dose reduction techniques available, these were not used during this study so we could systematically control $\mathrm{kV}$ and $\mathrm{mAs}$ for each scan.

\section{Conclusion}

This study demonstrates that while increased energy settings improve the visualization of certain anatomical structures, a threshold where increased radiation does not increase the acceptability of the scans occurs. Nearly a $50 \%$ reduction in radiation was possible while maintaining unanimous consensus among the evaluators that scans were suitable for surgery. This suggests that rhinologists should collaborate with radiologists to maximize the quality of information while reducing radiation exposure for our patients.

Additionally, our study does not demonstrate an improvement in visualization using IR techniques. Further advances in computational image processing will hopefully allow lower doses of radiation with improved visualization in the future.

\section{Funding}

FCL was supported by the National Institutes of Health grant 1UL1TR001111.

\section{Authorship contribution}

AJK: concept, data analysis, manuscript preparation; SWM: concept, data collection, data analysis, manuscript preparation; $\mathrm{BYH}$ : concept, data collection, data analysis, manuscript preparation; SBS: data collection, data analysis, manuscript preparation; BWM: data collection, data analysis, manuscript preparation; MT: data collection, data analysis; FCL: data analysis, manuscript preparation; CSE: data collection, data analysis; BAS: concept, data collection, data analysis, manuscript preparation.

\section{Availability of data and materials}

The datasets used and/or analysed during the current study are available from the corresponding author on reasonable request.

\section{Conflict of interest}

The authors declare that they have no competing interests.

\section{References}

1. Anand VK. Epidemiology and economic impact of rhinosinusitis. Ann Otol Rhinol Laryngol Suppl. 2004 May;193:3-5.

2. Orlandi RR, Kingdom TT, Hwang PH, et al. International Consensus Statement on Allergy and Rhinology: Rhinosinusitis. Int Forum Allergy Rhinol 2016; 6 Suppl 1:S22209.

3. Stevens WW, Lee RJ, Schleimer RP, Cohen NA. Chronic rhinosinusitis pathogenesis. J Allergy Clin Immunol 2015; 136:1442-1453.

4. Fokkens WJ, Lund VJ, Mullol J, et al. EPOS 2012: European position paper on rhinosinusitis and nasal polyps 2012. A summary for otorhinolaryngologists. Rhinology 2012; 50:1-12.

5. Chen JX, Kachniarz B, Gilani S, Shin JJ. Risk of malignancy associated with head and neck CT in children: a systematic review. Otolaryngol Head Neck Surg 2014; 151:554566.

6. Yuan MK, Tsai DC, Chang SC, et al. The risk of cataract associated with repeated head and neck CT studies: a nationwide populationbased study. AJR Am J Roentgenol 2013;

\section{1:626-630.}

7. Doss M. Conclusion of increased risk of cataracts associated with CT studies of the head may not be justified. AJR Am J Roentgenol 2014; 202:W413.

8. Herman GT, Herman GT. Fundamentals of computerized tomography: image reconstruction from projections. Dordrecht; New York: Springer, 2009.

9. Schulz B, Beeres M, Bodelle B, et al. Performance of iterative image reconstruction in CT of the paranasal sinuses: a phantom study. AJNR Am J Neuroradiol 2013; 34:1072-1076

10. Cicchetti D. Guidelines, Criteria, and Rules of Thumb for Evaluating Normed and Standardized Assessment Instrument in Psychology. Psychological Assessment 1994; 6:284-290.

11. Jessen KA, Shrimpton PC, Geleijns J, et al. Dosimetry for optimisation of patient protection in computed tomography. Appl Radiat Isot 1999; 50:165-172.

12. Setzen G, Ferguson BJ, Han JK, et al. Clinical consensus statement: appropriate use of computed tomography for paranasal sinus disease. Otolaryngol Head Neck Surg 2012; 147:808-816.

Brent A. Senior, MD

Department of Otolaryngology -

Head and Neck Surgery

University of North Carolina

Memorial Hospitals

170 Manning Dr.

Ground Floor Physician Office

Building

CB\#7070, Chapel Hill

NC 27599

USA

Tel: +1-919-966-3342

Fax: +1-919-966-7941

E-mail: brent_senior@med.unc.edu 